\title{
Desenvolvimento e avaliação de um protótipo de cortador de base para colhedoras de cana-de-açúcar ${ }^{1}$
}



Carlos E. S. Volpato ${ }^{2}$, Oscar A. Braunbeck ${ }^{3}$ \& Carlos A. A. de Oliveira ${ }^{4}$

\footnotetext{
1 Trabalho extraído da Tese de Doutorado do primeiro autor

2 DEG-UFLA. CP 37, CEP 37200-000, Lavras, MG. Fone: (35) 3829-1669. E-mail: volpato@ufla.br (Foto)

${ }^{3}$ FEAGRI-UNICAMP. CEP 13083-970, Campinas, SP. Fone: (19) 3788-1049. E-mail: oscar@agr.unicamp.br

4 FEAGRI-UNICAMP. CEP 13083-970, Campinas, SP. E-mail: caaoliv@agr.unicamp.br
}

Protocolo $131-14 / 9 / 2001$

\begin{abstract}
Resumo: Um protótipo de cortador de base para o seguimento pantográfico do perfil em colhedoras de cana-de-açúcar, foi projetado e construído e seu desempenho de flutuação foi avaliado em função da força normal de reação do perfil $\left(F_{N}\right)$. O trabalho foi conduzido em uma caixa de solo, o protótipo montado sobre um carro porta-ferramentas e submetido a ensaios nos quais variaram três tipos de perfil: senoidal, rampa ascendente e rampa descendente, duas amplitudes do perfil, 0,025 e 0,05 m e cinco velocidades de deslocamento: 0,69, 1,12, 1,48, 1,82 e 2,12 $\mathrm{m} \mathrm{s}^{-1}$. Os melhores resultados foram verificados para os perfis em rampa, tanto ascendente quanto descendente, independentemente da amplitude do perfil e da velocidade de deslocamento e, também, para perfil senoidal de ambas as amplitudes, com velocidade de deslocamento inferior a $1,48 \mathrm{~m} \mathrm{~s}^{-1}$. Os resultados demonstraram que o protótipo ainda é passível de ajustes, uma vez que o desempenho de flutuação sobre o perfil pode ser melhorado através da utilização de técnicas de otimização.
\end{abstract}

Palavras-chave: cana-de-açúcar, colhedora, cortador de base, seguimento pantográfico

\section{Development and evaluation of a prototype of base cutter for sugar-cane harvesters}

\begin{abstract}
A prototype of base cutter for sugar-cane harvester was designed and built. The floating performance was evaluated as a function of the profile reaction force $\left(\mathrm{F}_{\mathrm{N}}\right)$. The prototype was tested in a soil box using a carrier car running on sinusoidal and ramp type profiles with amplitudes of 0.025 and $0.05 \mathrm{~m}$. The travel speeds used were of five different levels: 0.69 , $1.12,1.48,1.82$ and $2.12 \mathrm{~m} \mathrm{~s}^{-1}$. The best results were obtained while running on ramp profiles, both ascending or descending, independent of the profile amplitude or the travel speed. The sinusoidal profile showed good results for the travel speed of $1.48 \mathrm{~m} \mathrm{~s}^{-1}$, for both the amplitudes tested. The results demonstrated that the prototype may still be adjusted, as the floating action on the profile can be improved through the use of optimization techniques.
\end{abstract}

Key words: sugar-cane, harvester, base cutter, pantographic following

\section{INTRODUÇÃO}

As perdas e a contaminação da matéria-prima com terra durante o processo de colheita, estão associadas ao não acompanhamento pantográfico do perfil do solo pelo cortador de base das colhedoras (Volpato, 2001).

Os cortadores de base atualmente utilizados nas colhedoras de cana-de-açúcar e forragens, estão rigidamente fixados à estrutura da máquina sem recursos adequados para $o$ seguimento do perfil do solo, apesar do conjunto cortador basal possuir acionamento hidráulico, permitindo variação na altura do corte para cortar colmos em um ponto um pouco acima do nível do terreno, ou seja, a touceira deverá estar sobre um leve camalhão.

Segundo Ripoli \& Paranhos (1990) esta condição é raramente encontrada, pois as práticas de preparo do solo e plantio em uso no Brasil ainda não estão totalmente voltadas para a colheita mecanizada. Desta forma, quando a cana-deaçúcar se encontra em sulcos mais profundos, corta-se um grande volume de solo, correndo-se o risco da soqueira ser destruída durante o corte e, quando a cana se encontra dentro do sulco, não há possibilidade da máquina cortar na altura desejada, havendo mais dilaceramento do internódio que, propriamente, um cisalhamento, gerando um corte não 
homogêneo. Esta condição de corte deixa um toco muito alto, aumentando as perdas de campo; aumenta a probabilidade de ataque de insetos e doenças, devido aos danos gerados na soqueira remanescente; provoca danos nas lâminas das colhedoras, diminuindo os intervalos de manutenção e, por fim, provoca diminuição da qualidade de colheita, ao incorporar terra à matéria-prima colhida.

Essas condições promovem perdas em quantidade e qualidade, pela contaminação do mesmo com terra e microrganismos do solo, além de aumentar o custo de processamento na usina. Segundo Magalhães \& Braunbeck (1999) perdas de matéria-prima podem chegar, em certos casos, a mais de $10 \%$, e o recolhimento de terra, pela colhedora, é da ordem de 3 a $5 \mathrm{~kg} \mathrm{t}^{-1}$ de matéria-prima colhida.

Segundo Ridge (1990) uma das formas de minimizar o problema da presença de solo na matéria-prima está relacionada às alterações nas práticas culturais existentes (preparo do solo, plantio, largura entre fileiras etc.) ou através de mudanças no projeto do disco cortador basal. Segundo Ridge \& Dick (1992) os fatores relacionados ao corte basal e que contribuem para o alto nível de solo na matéria-prima, são ação de corte e movimentação de solo (efeito aração) das sapatas dos levantadores helicoidais de cana deitada; excessivo ângulo empurrador de colmo antes do corte basal e excessivo fluxo de solo no cortador basal, quando o corte é realizado abaixo do nível do solo.

Segundo Braunbeck (1999) existem limitações no princípio operacional do cortador de discos rígidos, gerando um espaço tecnológico para o dimensionamento de mecanismos alternativos que diminuem ou evitam o recolhimento de solo e as perdas de matéria-prima durante a colheita.

Assim sendo, este trabalho teve como objetivo dimensionar um mecanismo articulado de quatro barras, capaz de acompanhar a superfície do perfil de solo com base na força de interação desenvolvida no ponto de contato solo-disco.

\section{MATERIAL E MÉTODOS}

\section{Projeto do cortador de base}

O projeto do mecanismo de quatro barras foi feito em computador digital, utilizando-se o programa para desenho mecânico AutoCad R-14® e Mechanical Desktop ${ }^{\circledR}$, versão 2.0. Suas partes constituintes foram dimensionadas em função das características operacionais de uma colhedora de cana-deaçúcar acoplada a trator agrícola de rodas, em sistema de cana crua e inteira, colhendo-se uma linha de cana por passada. A construção e a montagem física do protótipo foram feitas pela empresa USITEP, de Piracicaba, SP, enquanto as peças foram construídas utilizando-se, como material base, o ferro fundido e chapas de aço.

\section{Local de realização dos ensaios}

Os ensaios foram realizados em uma caixa de solo localizada no Laboratório de Protótipos do Departamento de Máquinas Agrícolas da FEAGRI/UNICAMP. A caixa de solo utilizada nos ensaios apresentava dimensões de $15 \mathrm{~m}$ de comprimento, $2 \mathrm{~m}$ de largura e 1,5 $\mathrm{m}$ de profundidade. O protótipo do cortador de base foi montado em um carro porta-ferramentas, que é uma estrutura tipo pórtico, com quatro rodas-guia nos seus pontos de apoio, que trafegam sobre trilhos dispostos ao longo das laterais da caixa de solo, cujas funções são de posicionamento, sustentação, tração e controle, e se encontram descritas na Figura 1.

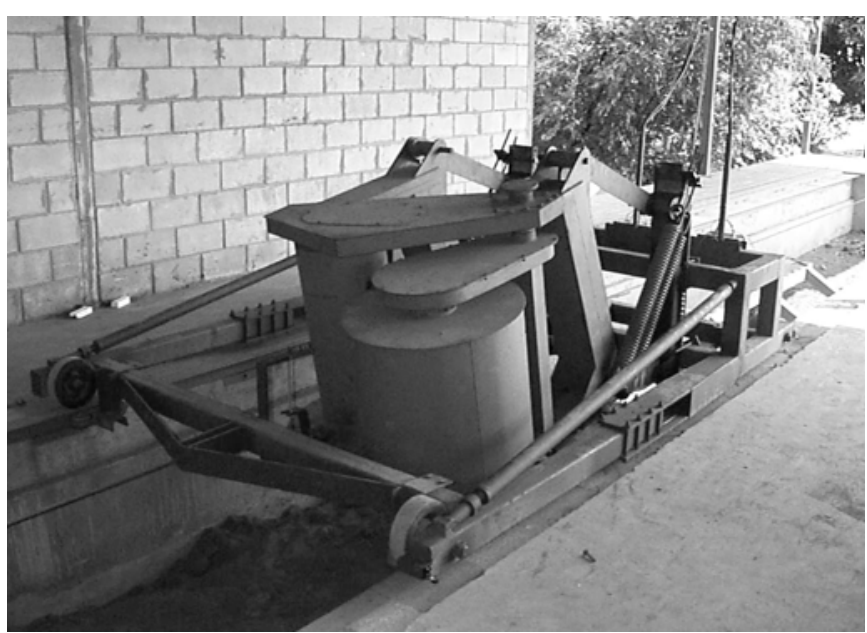

Figura 1. Cortador de base montado no carro porta-ferramentas sobre a caixa de solo

O carro porta-ferramentas foi tracionado por um cabo de aço de $1 / 2$ " de diâmetro, o qual é acionado por um tambor rotativo, solidário a um motor hidráulico localizado em um fosso anexo à caixa de solo; este motor é parte de uma transmissão hidrostática com velocidade variável, cuja bomba de deslocamento variável está acoplada a um motor diesel estacionário, com potência de $22 \mathrm{~kW}$. A Figura 2 mostra o fluxograma de acionamento do conjunto acima.

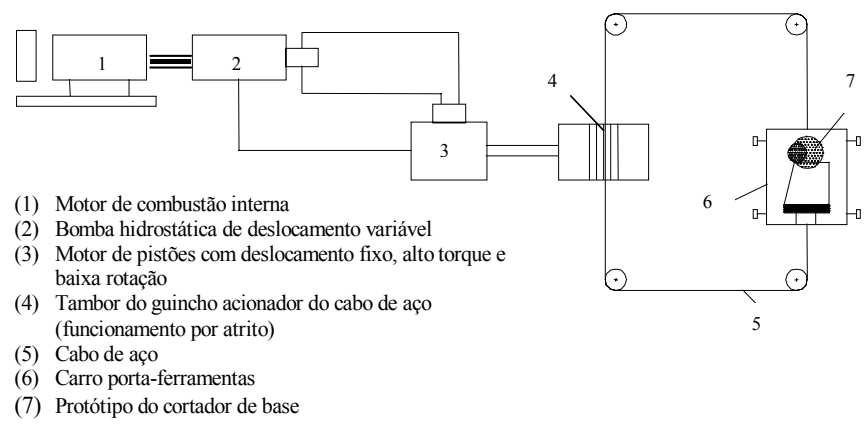

Figura 2. Fluxograma de acionamento do carro porta-ferramentas

\section{Parâmetros variados durante os ensaios}

Durante os ensaios foram variados os seguintes parâmetros experimentais: tipo de perfil (senoidal, rampa ascendente e rampa descendente), amplitude do perfil $(0,025$ e $0,05 \mathrm{~m}) \mathrm{e}$ velocidades de deslocamento do carro porta-ferramentas $(0,68$, $1,12,1,48,1,82$ e $\left.2,20 \mathrm{~m} \mathrm{~s}^{-1}\right)$.

\section{Coleta de dados}

Os dados registrados e coletados durante os ensaios foram força de interação do disco com o perfil, distância entre o perfil e o disco e os pulsos gerados pelo cabeçote indutivo. A força de interação entre disco e perfil foi medida através de uma 
célula de carga GEFRAN®, com capacidade de $5.000 \mathrm{~N}$ e sensibilidade de $3 \mathrm{mV} \mathrm{V}^{-1}$, fixada no extremo frontal inferior do disco de corte basal. A distância entre o perfil e o disco foi medida através de um transdutor linear de deslocamento (LVDT) GEFRAN ${ }^{\circledR}$, com capacidade de até $150 \mathrm{~mm}$, fixado na extremidade frontal do mecanismo, próximo à célula de carga. Os dados acima descritos, em forma de sinais, foram armazenados por um computador portátil, após tratamento dos sinais em um equipamento de aquisição de dados denominado "Spidder". Para melhor entendimento do sistema de aquisição dos dados, elaborou-se um fluxograma que se encontra ilustrado na Figura 3.

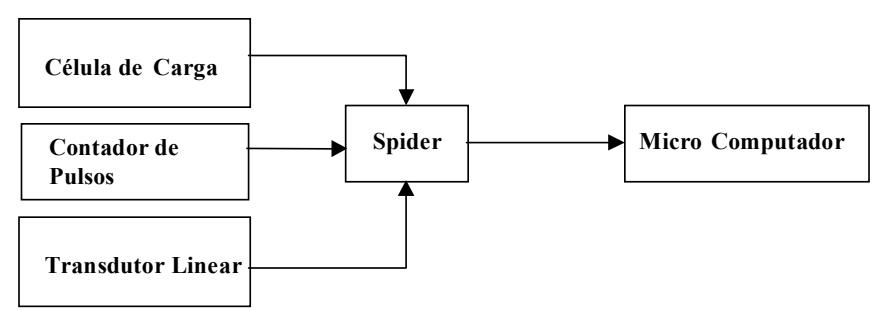

Figura 3. Fluxograma para aquisição dos dados

Realizaram-se cinco aquisições com uma repetição para cada situação. Como existiam 30 situações, então foram feitas 300 aquisições. Os dados foram adquiridos na taxa de aquisição de $200 \mathrm{~Hz}$. Após o término dos ensaios, os dados foram tratados pelo software Excel do Windows ${ }^{\circledR}$ para geração das planilhas de trabalho e dos gráficos de saída.

\section{Análise do desempenho de flutuação do mecanismo}

O desempenho de flutuação do mecanismo cortador de base foi avaliado em função do comportamento da força normal de reação do perfil, onde se observou, para todos os ensaios, seus valores máximos, mínimos, médios e a amplitude desta força.

\section{RESULTADOS E DISCUSÃO}

Os resultados das repetições para um mesmo ensaio obtiveram, praticamente, os mesmos valores, sendo consideradas estatisticamente iguais para todos os níveis de significância. Por se tratar de um sistema mecânico, com todas as variáveis controladas e sem influência externa capaz de provocar alguma alteração no ambiente de aquisição, esta análise foi totalmente coerente com o esperado e, portanto, plenamente justificada. Desta forma, os resultados apresentados para análises e discussões foram obtidos a partir de um único ensaio para cada situação, conforme mostra a Tabela 1.

Através da Tabela 1, pode-se analisar o comportamento da força normal de reação do perfil para os três tipos de perfil avaliados. Observa-se que a força mínima $\left(\mathrm{F}_{\mathrm{MIN}}\right)$ registrada para quase todos os ensaios, foi zero (0) $\mathrm{N}$ significando que pelo menos em um ponto ao longo do perfil houve perda de contato entre a ponta do disco cortador de base e o perfil, cuja célula de carga registrou força igual a zero.

Os resultados para perfil senoidal mostram que a amplitude do perfil e a velocidade de deslocamento da máquina têm
Tabela 1. Características da força normal de reação para perfil do tipo senoidal (A), rampa ascendente (B) e rampa descendente (C) em função da velocidade de deslocamento (v) e da amplitude do perfil (AMP)

\begin{tabular}{|c|c|c|c|c|c|}
\hline & $\mathrm{v}\left(\mathrm{m} \mathrm{s}^{-1}\right)$ & $\mathrm{F}_{\operatorname{MAX}}(\mathrm{N})$ & $\mathrm{F}_{\mathrm{MIN}}(\mathrm{N})$ & $\mathrm{F}_{\mathrm{MED}}(\mathrm{N})$ & $\mathrm{F}_{\mathrm{AMP}}(\mathrm{N})$ \\
\hline \multicolumn{6}{|c|}{$\mathrm{AMP}=0,025(\mathrm{~m})$} \\
\hline \multirow{6}{*}{ A } & 0,69 & 1585,41 & 0 & 593,11 & 1585,41 \\
\hline & 1,12 & 1812,38 & 0 & 590,28 & 1812,38 \\
\hline & 1,48 & 2410,22 & 0 & 583,69 & 2410,22 \\
\hline & 1,82 & 2502,13 & 0,79 & 573,63 & 2502,13 \\
\hline & 2,12 & 3403,22 & 0 & 543,84 & 3403,22 \\
\hline & 0,69 & 1048,78 & 0 & 438,45 & 1048,78 \\
\hline \multirow{4}{*}{$\mathrm{B}$} & 1,12 & 1253,59 & 0 & 364,18 & 1253,59 \\
\hline & 1,48 & 1374,24 & 0 & 358,15 & 1374,24 \\
\hline & 1,82 & 1406,45 & 0 & 410,00 & 1406,45 \\
\hline & 2,12 & 1541,12 & 0 & 493,98 & 1541,12 \\
\hline \multirow{5}{*}{$\mathrm{C}$} & 0,69 & 1146,78 & 0 & 411,28 & 1146,78 \\
\hline & 1,12 & 1389,87 & 0 & 404,18 & 1389,87 \\
\hline & 1,48 & 1489,50 & 0 & 493,05 & 1489,50 \\
\hline & 1,82 & 1516,45 & 0 & 300,07 & 1506,45 \\
\hline & 2,12 & 1601,12 & 0 & 322,73 & 1601,12 \\
\hline \multicolumn{6}{|c|}{$\mathrm{AMP}=0,05(\mathrm{~m})$} \\
\hline \multirow{5}{*}{ A } & 0,69 & 3794,52 & 0 & 969,02 & 3794,52 \\
\hline & 1,12 & 4128,33 & 0 & 962,19 & 4128,33 \\
\hline & 1,48 & 4817,30 & 0 & 971,08 & 4817,30 \\
\hline & 1,82 & 4919,29 & 1,57 & 963,73 & 4919,29 \\
\hline & 2,12 & 6462,35 & 0 & 1205,84 & 6462,35 \\
\hline \multirow{5}{*}{ B } & 0,69 & 2934,49 & 0 & 960,07 & 2934,49 \\
\hline & 1,12 & 3288,27 & 0 & 546,68 & 3288,27 \\
\hline & 1,48 & 3593,85 & 0 & 488,33 & 3593,85 \\
\hline & 1,82 & 3756,51 & 0 & 761,39 & 3756,51 \\
\hline & 2,12 & 4084,06 & 0 & 813,63 & 4084,06 \\
\hline \multirow{5}{*}{$\mathrm{C}$} & 0,69 & 2825,49 & 0 & 871,19 & 2825,49 \\
\hline & 1,12 & 3211,27 & 0 & 665,68 & 3211,27 \\
\hline & 1,48 & 3606,85 & 0 & 671,33 & 3606,85 \\
\hline & 1,82 & 3703,51 & 0 & 803,39 & 3703,51 \\
\hline & 2,12 & 3984,06 & 0 & 878,63 & 3984,06 \\
\hline
\end{tabular}

influência significativa sobre o comportamento da força normal de reação do perfil $\left(\mathrm{F}_{\mathrm{N}}\right)$. Este comportamento se dá pelo fato desta amplitude de força corresponder à componente inercial, que movimenta a massa do cortador de base, ou seja, surge da aceleração desta massa, a qual é proporcional à amplitude do perfil e à velocidade angular. Para os perfis em rampa, tanto ascendente quanto descendente, a velocidade de avanço da máquina demonstrou menor influência sobre variação da força normal de reação do perfil justificando-se, assim, pelo fato da componente vertical de aceleração ser nula.

A amplitude do perfil teve muita influência sobre a amplitude da força normal de reação do perfil, visto que, quando seu valor é dobrado, isto é, passando de 0,025 para $0,05 \mathrm{~m}$, observase que a amplitude da força aumenta nesta proporção, independentemente do valor da velocidade de deslocamento da máquina e do tipo de perfil.

Em relação desempenho de flutuação (seguimento do perfil) do mecanismo cortador de base, as melhores condições verificadas foram para os perfis em rampa, tanto ascendente quanto descendente, independentemente da amplitude do perfil e da velocidade de deslocamento, e para perfil senoidal de ambas as amplitudes com velocidade de deslocamento inferior 
a $1,48 \mathrm{~m} \mathrm{~s}^{-1}$. Este bom desempenho de flutuação pode ser observado na Figura 4, na qual se percebe o pouco ou quase nenhum registro de força igual a zero. As piores condições ocorrem para o perfil senoidal com velocidade de deslocamento da máquina superior a $1,88 \mathrm{~m} \mathrm{~s}^{-1}$ para ambas as amplitudes do perfil. Neste caso, fica nítida a tendência de afastamento do cortador de base do perfil, conforme se pode verificar através da Figura 5.

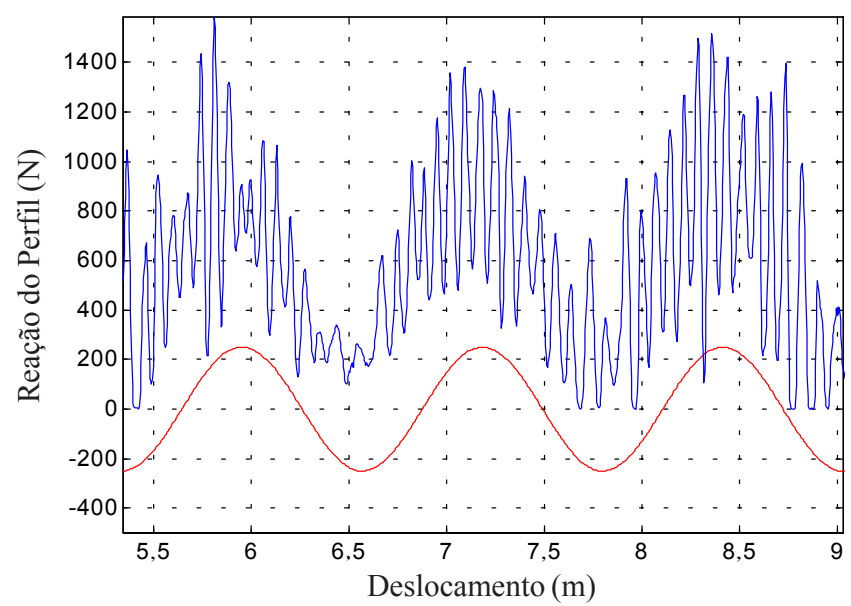

Figura 4. Força normal de reação $\left(\mathrm{F}_{\mathrm{N}}\right)$ para perfil senoidal de amplitude de $0,025 \mathrm{~m}$ e velocidade de deslocamento de $0,69 \mathrm{~m} \mathrm{~s}^{-1}$

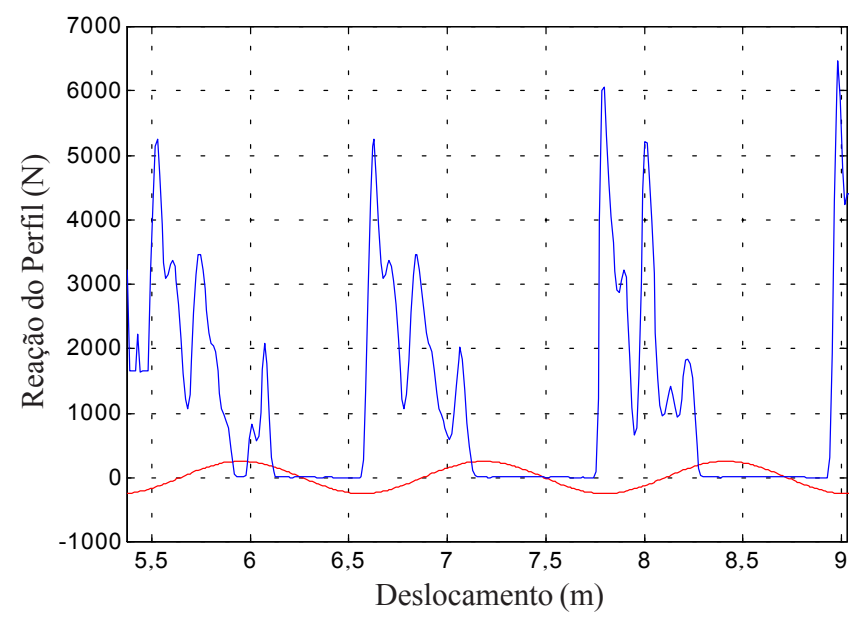

Figura 5. Força normal de reação $\left(\mathrm{F}_{\mathrm{N}}\right)$ para perfil senoidal de amplitude de $0,05 \mathrm{~m}$ e velocidade de deslocamento de 2,12 $\mathrm{m} \mathrm{s}^{-1}$

Por intermédio da Figura 4 percebe-se que, para este caso, o desempenho de flutuação foi considerado bom porque a presença de forças iguais a zero são pouco freqüentes, o que garante contato contínuo entre o mecanismo e o perfil; já para a situação representada pela Figura 5, observa-se que o mecanismo freqüentemente se afasta do perfil, principalmente na parte descendente da senóide, mostrando desempenho ruim de flutuação, nesta condição.

Uma avaliação mais aprofundada sobre a tendência de penetração do disco no solo fica prejudicada, em razão da impossibilidade deste trabalho em quantificar tal fator; entretanto, através da força média mostrada na Tabela 1, tem-se uma primeira referência a respeito. Observa-se que, para todas as condições de ensaio, o valor médio mínimo registrado foi de
$300 \mathrm{~N}$ e o valor médio máximo foi de $1205 \mathrm{~N}$. Especificamente para esse caso, em que o mecanismo cortador de base foi projetado com sistema de seguimento do perfil passivo, isto é, o mecanismo é posicionado nos movimentos ascendentes, pela força de reação do solo e, nos movimentos descendentes, pela ação do próprio peso, cujos valores podem ser considerados altos, porém ainda passíveis de melhorias se se lhes aplicar um processo de otimização.

\section{CONCLUSÕES}

1. O protótipo do mecanismo cortador de base projetado para o seguimento pantográfico do perfil, obteve uma avaliação de razoável para boa, no que tange ao seu desempenho de flutuação.

2. O desempenho de flutuação do mecanismo foi sensivelmente influenciado pelos tipos de perfis trafegados.

3. De forma especial, o perfil senoidal apresentou-se como o mais crítico, uma vez que sofreu influência de todas as variáveis avaliadas.

4. O mecanismo de corte basal apresentou dificuldades de flutuação na parte descendente da senóide para velocidade superior a $1,48 \mathrm{~m} \mathrm{~s}^{-1}$ e para as duas amplitudes de perfil avaliadas.

5. Um futuro tratamento de otimização aplicado a este mecanismo, pode melhorar seu desempenho de flutuação ao minimizar a força de reação.

\section{AGRADECIMENTOS}

Os autores são extremamente gratos à FAPESP, pelo financiamento do projeto que originou este trabalho, e à empresa USITEP, de Piracicaba, por julgar que esta acredita no desenvolvimento tecnológico da engenharia agrícola ao construir e se interessar pelo desenvolvimento da tecnologia desenvolvida por este projeto.

\section{LITERATURA CITADA}

Braunbeck, O.A. Proposta brasileira de colheita mecanizada. In: Seminário soluções e novidades na mecanização da canade-açúcar, 1, 1999, Ribeirão Preto. Anais... Ribeirão Preto: IDEA, 1999. p. 56-60.

Magalhães, P.S.G.; Braunbeck, O.A. Colheita de cana-deaçúcar: Atualidade e perspectiva. In: Congresso de Ingeniería Rural y Mecanización Agraria en el Ambito Latinoamericano, 3, 1998, La Plata. Anais... La Plata: ALIA, 1998. p.262-271.

Ridge, D.R. Minimizing the problem of soil in chopper harvester cane. Proceeding of the International Society of Sugarcane Technologists, Kuala Lumpur, v.16, p.315-323, 1990.

Ridge, D.R.; Dick, R.G. Soil intake with cane during chopper harvesting: Minimising the problem. Proceedings of Australian Society of Sugar Cane Technologists. Quennsland, v.14, p.25-30, 1992.

Ripoli, T.C.; Paranhos, S.B. Máquinas para corte e carregamento de cana. Piracicaba: Centro Acadêmico Luiz de Queiroz, 1990. 46p. Departamento Editorial

Volpato, C.E.S. Otimização de um cortador de base para seguimento do perfil de solo em colhedoras de cana-deaçúcar. Campinas: UNICAMP, 2001. 185p. Tese Doutorado 